Aus der chirurgischen Universitätsklinik zu Leipzig.

(Direktor: Geh. Med.-Rat Prof. Dr. E. Payr.)

\title{
Die Hammerzehe.
}

Von Dr. Karl Schläpfer, gew, Assistent der Klinik.

(Mit 2 Abbildungen.)

Unter den Zehendeformitäten, welche in einer nicht geringen Anzahl von schwereren Fällen zweckentsprechende Behandlung erheischen, gehört die $\mathrm{Hammerzehe} \mathrm{(hammertoe}$ [A d a $\mathrm{m} \mathrm{s}$ ], orteil en marteau).

Von französischer Seite ( $R$ o u s s e lot) ist schon in der zweiten Hälfte des 18. Jahrhunderts auf diese Deformität hingewiesen worden. Auch in der Folge (Boyer, Blandin, Blum, Cohen, Dubreuil, Debaussaux, Malgaigne, Néla. ton, Petrequin, Roche et Sanson) nahm sie in der franz. Literatur einen breiten Raum ein. A da ms hat I 892 den Begriff der Hammerzehe klinisch genauer so umschrieben, wie er jetzt noch Gültigkeit hat. Besonders Nicoladoni hat sich eingehend mit diesem Krankheitsbilde beschäftigt. Eichenwald, Hoffa, Hoffmann, Kirsch, Ranneft, Rubritius und Vulpi us trugen mit zum Ausbau der Klinik, bzw. Pathogenese teils der Therapie der Hammerzehe bei. In der jüngsten Zeit finden wir in der Literatur nur wenige Abhandlungen über diese Mißbildung. Daher hielt ich es für angezeigt, die bisher in der Literatur niedergelegten Anschauungen zusammen mit den an hiesiger Klinik im Laufe der Zeit gewonnenen Erfahrungen darzulegen, um im Anschlusse daran eine neue, von Herrn Geheimrat Pay r eingeführte chirurgische Behandlungsmethode mitzuteilen.

Die Hammerzehe ist eine selten angeborene, meistens erworbene, allmählich entstandene Flexionskontraktur im I. Interphalangealgelenke 
einer oder mehrerer Zehen; in der Regelder zweiten $Z$ ehe (Adams).

Bevor wir den vorliegenden Begriff der Hammerzehe analysieren, müssen wir uns kurz die Anatomie und Physiologie der Zehen vergegenwärtigen, soweit uns dieselben zum richtigen Verständnisse des klinischen Bildes nötig sind. Betrachten wir zuerst die Art der gelenkigen Verbindung der Phalangen untereinander und zu den Metatarsi: Es sind ausgesprochene Scharniergelenke, welche daher nur Beugung und Streckung zulassen. Während die Metatarsophalangeal- und I. Interphalangealgelenke normale Verhältnisse aufweisen: Knorpelig überzogene Gelenkflächen an entsprechend geformten Knochenenden, Gelenkkapsel mit Verstärkungsbändern, sind die übrigen Interphalangealgelenke, speziell der kleinen Zehen, rudimentär ausgebildet, in 36 Proz. der Fälle nach $\mathrm{Me} \mathrm{r} \mathrm{k} \mathrm{e} \mathrm{l} \mathrm{sogar} \mathrm{synostotisch} \mathrm{miteinander}$ verbunden. Da die Gelenkveränderungen bei der Hammerzehe pathognomonisch sein können, möge der Bau des normalen I. Interphalangealgelenks im allgemeinen kurz skizziert werden. Dabei halte ich mich an die Ausführungen von Merkel: Die Basis der Trochlea der Grundphalanx (distales Ende) ist sanduhrförmig gebaut, ähnlich der Trochlea des Ellbogens. Die Basis der Mittelphalanx (proximaler Anteil) weist eine entsprechende Krümmung mit sehr schwacher Höhlung auf. An der Trochlea findet man den dickern Knorpelüberzug als an dem zugehörigen Pfannenanteile. Die Kapsel setzt sich an der Volarseite der Rolle etwas weiter vom Knorpelrande entfernt an. In das Innere des Gelenks springen zwei Synovialfalten vor, kleine Inkongruenzen der Gelenkflächen ausgleichend. Auf der Dorsalseite zeigt die Gelenkkapsel enge Verbindung mit der Strecksehne. Eine nicht unwichtige Rolle spielen die Verstärkungsbänder des Gelenks. Als besonders wichtig für die Ätiologie der Hammerzehe erweisen sich die Ligamenta collateralia. Sie setzen proximal an den Gruben und den dorsal davon liegenden Höckern an den Seiten der Metatarsal- und Tarsalköpfchen an und heften sich an entsprechend gebildeten Höckern der Basis der Grund- bzw. Mittelphalangen an. Leichenuntersuchungen, die Herr Geheimrat $\mathrm{Payr}$ schon vor Jahren vornahm, ergaben, daß die Mehrzahl der Fasern der Ligamenta collateralia plantar bzw. hinter der durch die Mitte 
der Knochen von Zehe-Metatarsus gezogenen Längsachse (in Streckstellung) liegen. Schon bei leichter Flexion des Gelenks kommt ein noch größerer Teil des Ligamentum collaterale an die Beugeseite zu liegen. Mit einzelnen Fasern gehen diese Bänder plantar in die transversal verlaufenden Ligamenta accessoria über. Teils hängen Fasern mit den in die Zehen ausstrahlenden Zipfeln der Aponeurosis plantaris zusammen. Die Lage der Ligamenta collateralia und deren Beziehung zu der Plantaraponeurose wird uns weiter unten bei der $\ddot{A}$ tiologie der Hammerzehe wichtige Gesichtspinkte eröffnen.

Länge, Form und Haltung der Zehen beim Gehen muß uns gegenwärtig sein, wenn wir uns ein Bild von den bei der Hammerzehe unausbleiblichen Veränderungen, sowie deren Zusammenhang mit dem Gehakte machen wollen. Dabei haben wir Füße im Sinne, die nicht durch Tragen von zu engen Schuhen bereits deformiert sind. Normalerweise überragt die zweite Zche die erste und dritte um Weniges. Gegen die Spitze zu nehmen die Zehen an Umfang zu und endigen in der beim Gehakte wichtigen Zehenbeere: Einem durch subkutane Fettablagerung verdickten und kräftigen Polster. Die Großzehe zeigt noch die größte Ähnlichkeit mit dem Daumen. Die Beere ist am wenigsten auf das Ende lokalisiert. Deutlich ausgesprochen findet sich diese terminale, kolbige Verdickung dagegen an den übrigen Zehen. Leicht plantar (krallenartig) gebeugt werden die zweite bis fünfte Zehe normalerweise gehalten. Beim Abstoßen des Fußes mit der Fußspitze werden die krallenartig gebogenen Zehenbeeren mit den vordern Ballen gegen den Boden angestemmt. Dadurch wird dem Schritte, wie Merkel dartut, seine Sicherheit verliehen. Anfänglich zeigen. die Zehen rundliche Form. Durch das Tragen von Schuhen platten sie sich mit den Seiten, an denen sie aneinanderliegen, gegen. einander $\mathrm{ab}$. In stärkeren. Graden von beengenden Schuhen werden die Zehen übereinander verschoben. Die Großzehe wird lateralwärts gedrückt. Sie liegt entweder über oder unter der zweiten Zehe. Indem auch die dritte.Zehe medialwärts unter die zweite Zehe sich drängt, wird die zweite Zehe dorsalwärts verlagert. Dadurch kann, wie wir später noch sehen werden, cine Vorbedingung zur Entstehung der Hammerzehe geschaffen werden. Wenn dic Zehen teilweise übercinanderliegen und gleichzeitig seitliche 
Verkrümmungen aufweisen im Sinne der Abduktion, so haben wir die Anordnung der Zehen in zwei Lagen (chevauchement des orteils). Dabei werden die zweite und vierte Zehe meist zu Hammerzehen, indem speziell die zweite Zehe von vorn und von der Seite her gestaucht und somit ihrer Basis näher gerückt wird.

Nachdem wir die normale Anatomie und Physiologie der Zehengelenke soweit nötig gestreift haben, gehen wir zur Klinik und Pathogenese der Hammerzehe über. Nach Adams stellt, wie bereits erwähnt, die Hammerzehe eine selten angeborene, meist erworbene, allmählich entstandene Flexionskontraktur im $I_{i}$ Interphalangealgelenke einer oder gleichzeitig mehrerer Zehen dar. In der Regel ist nur die zweite Zehe betroffen. Nach Pay r kommt die Deformität nicht allzu selten angeboren vor. Es liegen Angaben über Heredität vor. Die Grundphalanx steht dorsal hyperextendiert, die zweite Phalanx in wechselndem Grade flektiert. In den stärksten Graden ist das Mittelglied gegenüber dem Grundgliede dorsal subluxiert. Das Zehenendglied steht entweder I. in der Richtung der zweiten Phalanx (L), oder 2. dorsal $(Z)$, oder 3 . plantar flektiert (C). Cohen hat diese Formen genau unterschieden. Den erstgenannten Typus nannte er: type en $L$ oder Schwanenhalszehe (orteil en cou de cygne) (Nélaton), den zweiten: type en $Z$. Beim dritten dem: type en $C$ tritt der Patient mit der Rückenseite des Endzehengliedes auf. Bei dieser Einteilung werden die Lagebeziehungen der einzelnen Phalangen zueinander verglichen. Außerdem hat aber $\mathrm{C}$ ohen die Deformität nach dem Grade der anatomischen Veränderungen in drei Stufen abgeteilt: $\mathrm{Zu}$ Anfang besteht Flexionskontraktur im I. Interphalangealgelenke. Passiv läßt sich die Zehe in ihre normale Streckstellung zurückbringen. Sich selbst überlassen, tritt die Verkrümmung wieder auf. Unter der chronischen Druckwirkung bei dieser Flexionshaltung im I. Interphalangealgelenke bildet sich dorsal ein Hühnerauge, darunter ein Schleimbeutel infolge der beständigen Verschiebung von Haut gegen knöcherne Unterlage. Als Folge von chronischen Entzündungsprozessen, die wir-später genauer analysieren werden, kann in der Folge Ankylose im Gelenke auftreten (zweite Phase nach $\mathrm{Coh}$ en). In der dritten. Periode finden sich dorsa! Fistelgänge als Folge von nach außen durchgebrochenen Abszedierungen im subkutan entstandenen Schleimbeutel 
bzw. aus dem Gelenke (Schleimbeutelfistel). Weil dieses Bild große Analogie zum klassischen Malum perforans pedis zeigt, wurde es von Dubreuil mal dorsal des orteils genannt.

Über die Häufigkeit des Leidens finden sich nur vereinzelte Angaben. Debaussaux nimmt 1,65 Fälle an unter 1000 Personen. Unter Männern soll die Hammerzehe nach dem gleichen Autor häufiger vorkommen, als bei Frauen. Nach Payr's Erfahrungen ist das Verhältnis umgekehrt. Eine diesbezügliche statistische Erhebung ließe sich an poliklinischem Materiale vornehmen.

In den meisten Fällen handelt es sich um Leute nach der Pubertät, die bestimmter Beschwerden wegen zum Arzte kommen und Hammerzehen aufweisen. Langsam hat sich das Leiden ausgebildet. Der Beginn reicht oft Jahre zurück. Auch angeboren soll diese Mißbildung vorkommen. Z war wird dies von $\mathrm{Rous}$. selot bestritten. Symmetrisches Auftreten an beiden $\mathrm{I}$ üßen soll für kongenitale Form sprechen, besonders aber das gleichzeitige Auftreten mit anderen Deformitäten.

Betrachten wir erst die Pathogenese der erworbenen Form: $\mathrm{Zu}$ enges $\mathrm{Sch}$ uhwerk wird immer wieder verantwortlich gemacht (Cohen, Couteaud, Malgaigne, Nélaton).

Wir müssen dabei mit $\mathrm{C}$ ohe $\mathrm{n}$ unterscheiden zu kurze und zu schmale Schuhe. Beide Mängel sind ätiologisch für das Entstehen der Hammerzehe von größter Wichtigkeit. Bei der Mehrzahl der sog. Kulturmenschen handelt es sich um das Tragen von zu schmalen Schuhen. Die Längsachse des Fußes geht normalerweise durch die zweite Zehe. Der beiderseitige Druck des zu schmalen Schuhes macht sich somit maximal auf die zweite Zehe bemerkbar. Die Zehe wird entweder gehoben; oder es wird die Großzehe über die zweite Zehe gedrückt (Borchardt). Beim Abrollen des Fußes wird im ersteren Falle die Zehenbeere der zweiten Zehe, die an sich die übrigen um Weniges überragt, von dorsal nach plantar und proximal gestaucht. Die Zehe wird in Beugestellung gezwungen. Auch wo die zweite Zehe unter die Großzehe gedrückt wird - es besteht hierbei gleichzeitig Hallux valgus -, wird dieselbe durch den Druck der Großzehe in Beugestellung im Interphalängealgelenke gedrängt und damit zur Hyperextension im Metatarsophalangealgelenke. Bei der herrschenden 
Damenschuhmode, wo die Absätze sehr hoch sind, lälit sich ein viel häufigeres Vorkommen der Hammerzehe konstatieren (P a y r). Es ist darauf zurückzuführen, daß der Fuß auf der sehr schrägen Fußachse nach vorn gleitet und daß dabei die zweite Zehe als die längste, die andern überragende von distal her gestaucht und in Beugestellung gedrückt wird. Tagtäglich während Monaten bis Jahren wiederholt sich diese durch das Schuhwerk bedingte Flexionskontraktur der zweiten Zehe. Auf der Konvexität der Krümmung (über I. Interphalangealgelenke) tritt unter dem chronischen Reiz eine lokale Verdickung der Haut ein. Langsam bildet sich ein Hühnerauge. Wirkt das primär auslösende Moment weiter (zu enger Schuh), so entsteht unter dem Hühnerauge ein Schleimbeutel als Resultat der steten Verschiebung von Haut

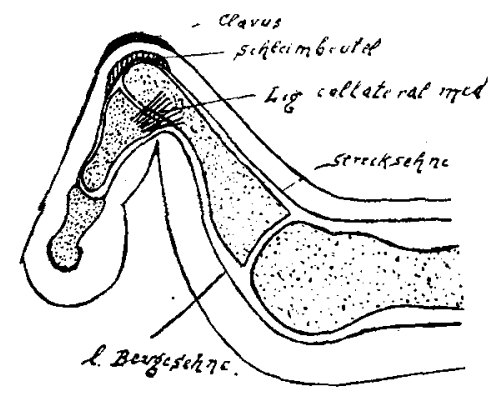

Fig. 1 .

gegen knöcherne Unterlage. Die chronische Reizung auf die Gewebe setzt sich auf das periartikuläre Gewebe fort: Es resultiert eine chronische Periarthritis. In den hochgradigsten Fällen finden wir die typischen Veränderungen einer Arthritis deformans. Die chronische Periarthritis bedingt eine Schrumpfung der Ligamenta collateralia und zugleich der Gelenkkapsel (Fig. I). Die Hammerzehe ist damit auch passiv nicht mehr zu redressieren, ohne Zerreißung der Bänder (zweite Phase nach Cohen). Durch den anhaltenden Druck wird der Zehennagel breiter und kürzer. Gleichzeitig wird auch das Endglied breitgedrückt. Die Haut verdickt sich auch da, und ist mechanischen Schädigungen ungleich häufiger ausgesetzt. Auch kleine accidentelle Wunden vereitern und geben Anlaß zur Bildung von Geschwüren und damit einhergehenden, schleichenden Phlegmonen. Vom Hühnerauge aus 
kann sich ab und $z u$ in dem darunter befindlichen Schleimbeutel eine Entzündung lokalisieren mit konsekutiver Abszedierung. Der AbszeB tritt entweder nach außen durch (Art von Selbstheilung). Es resultiert eine Fistel mit wenig Tendenz zu Heilung. Oder die Entzündung greift periartikulär tiefer, oft bis ins Gelenk; daneben unter der Haut weiter. Sehnen können sich wie beim „Wurm“ nekrotisch abstoßen. Der Gelenkknorpel wird aufgelöst. Das Gelenk wird deformiert. Dieses Bild, von Dubreuil zuerst beschrieben, wurde daher mit Recht mal dorsal des orteils genannt. Glücklicherweise treten diese schwersten Komplikationen - drittes Stadium nach Coh en - sehr seltẹn auf.

Die zu engen bzw. zu schmalen Schuhe als wichtigstes ätiologisches Moment erk]ären uns, weshalb die Hammerzehe an der zweiten Zehe am häufjgsten beobachtet wird. Zeigt sich an den übrigen Zehen (3. bis 5.) Hammerzehenbildung, so muß deren Genese eine andere sein. Es können $\mathrm{Na}$ rben an der Plan t ar seite diese Zehendeformität zur Folge haben (Schnittwunden, Verbrennung). Dabei handelt es sich um per secundam geheilte Wunden, bei denen der Infekt als chronische Entzündung in die Tiefe sich fortsetzt, entlang der Plantarfascie, so daß eine Schrumpfung nicht nur der Haut, sondern vornehmlich auch der Ligamenta collateralia eintritt. Um militärfrei zu werden, belieben nach Cohen Querschnitte auf der Plantarseite mit Verschorfung der Wunde zwecks Erzielung einer tiefgreifenden Narbe. Andere Drückeberger durchtrennten die Strecksehne. Die nun ihres Antagonisten beraubte Beugesehne bewirkte eine zunehmende Flexionskontraktur (Hammerzehe). Ein dritter Modus der artifiziellen Hammerzehenerzeugung ist derjenige mittels Umschnürung der Zehe durch ein Band. Dadurch wurde eine reaktive Entzündung in Haut und Subkutis angeregt, welche wiederum eine zunehmend kontrakte Narbe erzeugt.

Blandin gab als Ursache der Hammerzehenbildung eine schleichende Phlegmone der A poneurosis plantaris an. Bei den anatomischen Erläuterungen sahen wir, daß die Ligamenta collateralia einen gewissen Zusammenhang mit der Plantaraponeurose aufweisen. An der Fußsohle findet sich eine kleine Stichwunde, der weiterhin keine Beachtung geschenkt wird. Oberflächlich sub crustam geheilt geht die Entzündung längs der Plantaraponeurose 
weiter, ohne nennenswerte subjektive Beschwerden. Sie heilt allmählich. Als direkte Folge der chronischen Entzündung resultiert eine narbige Kontraktur der Plantaraponeurose und der mit ihr zusammenhängenden Ligamenta collateralia. Zwar erhob hiergegen Debaussaux den nicht unberechtigten Einwurf, weshalb die Kontraktur nur an einer Zehe auftrete. Für manche Fälle kommt diese Ätiologie sicherlich in Betracht. Namentlich bei angeborenen Formen scheint sie mir von Bedeutung zu sein.

Durch gewaltsames Redressement von Klumpfüßen kann die Plantarfascie teilweise zerrissen werden. Narbige Schrumpfung kann hinterher Hammerzehenbildung zur Folge haben.

B o y e $\mathrm{r}$ hat eine muskuläre Theorie aufgestellt, wonach durch Retraktion des Extensors eine Beugekontraktur auftrete; oft wirkten nach B o y e r Flexoren und Extensoren zusammen. Wieso nur eine Zehe dann ergriffen wird? Eine so umschriebene Muskelaffektion muß mit Recht bezweifelt werden.

Von französischer Seite wurde sodann behauptet, der Druck von der Seite (bei zu engem Schuhwerk) habe eine Atrophie der Interossei der zweiten Zehe zur Folge. Nun strecken die Interossei die Grundphalanx und beugen die zwei übrigen Phalangen. Weshalb entstünde aber nur eine so umschriebene Atrophie der Interossei einer Zehe?

Auch die Syphilis soll in seltenen Fällen (Muskelgumma, Paralyse [Cohen]) das auslösende Moment abgeben für Hammerzehenbildung.

DaB bei Arthritis deformans auch Hammerzehen auftreten können, ist erklärlich. Doch alsdann ist das klinische Bild ein anderes: Die Hammerzehe ist eine Folgererscheinung des konstitutionellen Leidens, das sich auch noch in andern Gelenken manifestieren wird. Knorpelige Einlagerungen in Muskelsehnen (Frano), wohl als Nebenbefunde anderweitiger Arthritis deformans, müssen als Zufälligkeiten von untergeordneter Bedeutung für die Genese der Hammerzehe betrachtet werden. Die Arthritis deformans soll nicht in unsere Untersuchung einbezogen werden. Jedoch sahen wir weiter oben beim mal dorsal des orteils - der dritten Stufe nach Cohen - daB in den hochgradigsten Fällen von Hammerzehen infolge Abszeßdurchbruch ins Zehengelenk schwere arthritische Veränderungen beobachtet werden. 
Wo die Hammerzehe angeboren auftritt, muß ein Entwicklungsfehler angenommen werden. Ätiologisch wichtig dürften da, wie schon erwähnt, schleichende intrauterine Entzündungen in der Planta pedis sein. Bei den angeborenen Hammerzehen gesellen sich meist noch anderweitige Mißbildungen hinzu. Angebliche Erblichkeit des Leidens wird man so auslegen müssen, daß Personen der gleichen Familie zu gleichen Entwicklungsstörungen disponieren (Mendel). Unmittelbar nach der Geburt wird der Deformität keinerlei Beachtung geschenkt. Erst wenn durch das häufigere Gehen Beschwerden sich einstellen, werden die Eltern stutzig. Oft beobachtet man dann das symmetrische Auftreten der Hammerzehe an beiden Füßen. Co u t e a u d traf die Hammerzehe bei einigen stets nacktfüßig gehenden, stark mit Syphilis durchseuchten und vielfach mit andern Mißbildungen der Glieder behafteten Stämmen der Hochebene von Madagaskar an.

Pathologisch-anatomische Befunde bei Hammerzehen nehmen einen geringen Raum ein in der Literatur. Übereinstimmend berichten die Autoren über sehr starke Variationen in dem Grade der Veränderungen. So fand Gosselin keine Veränderung. Fra no beschreibt einen Fall, wo das Gelenk frei war, sich dagegen in den Extensorensehnen Knorpelkerne vorfanden. Die Epidermis war verdickt; der subkutane Schleimbeutel kommunizierte mit dem Gelenke. In einem Falle fehlte auf der Gelenkoberfläche der Knorpelüberzug. S a 3 fand die Basis der Grundphalanx verdickt, die Gelenkfläche der Mittelphalanx verbreitert und deformiert. Blum berichtet von zwei Fällen, wo eine Luxation der Mittelphalanx gegenüber der Grundphalanx bestand. Der Kopf der Grundphalanx war atrophisch mit intaktem Knorpelüberzuge. Die Gelenkoberfläche der Mittelphalanx war verdickt, der Knorpelüberzug normal. Die Gelenkkapsel war in toto verdickt, namentlich an der Plantarseite. Dieser letztgenannte Befund wird bei der Therapie noch von Interesse sein. Auch Coh e n berichtet von Fällen mit Luxation im I. Interphalangealgelenke und geringen sonstigen pathologischen Veränderungen, währenddem in andern Fällen arthritische Veränderungen der Gelenk. oberfläche bestanden. In einem Falle berichtet $\mathrm{C}$ o h e $\mathrm{n}$ von einem subkutanen Schleimbeutel und einem zweiten unter der Extensorensehne. Gleichzeitig fanden sich arthritische Veränderungen 
der Gelenkfläche: Matte Oberflächen und Usuren. Terrier's Fall wies chronische Dermatitis und Hypertrophie des Stratum Malpighii, sowie der Subkutis auf. Richelot fand Arrosion des Knorpelüberzuges derart, daß stellenweise der Knochen frei lag. Letzterer zeigte die Erscheinungen einer Ostitis. Auch die Haut über und in der Nachbarschaft des Hühnerauges war hypertrophisch. Diese kasuistischen Mitteilungen zeigen, wie different die anatomischen Veränderungen bei der Hammerzehe sein können. Durch chronische Reizung der Haut entsteht erst eine Verdickung, die an der Stelle der größten mechanischen Insultes zu Hühneraugenbildung führt. In der Umgebung des Clavus ist, wie Terrier und Cohen nachwiesen, die Epidermis ebenfalls verdickt. Das Hühnerauge stellt eine Reaktion der Haut dar gegen den chronischen Druck. Aus gleicher Ursache bildet sich auch der subkutane Schleimbeutel, ev. sogar ein zweiter unter der Strecksehne (Cohen). In den stärkst ausgesprochenen Fällen finden wir die Veränderungen ähnlich wie bei Arthritis deformans: Usur des Knorpels, Verlust der glänzenden Gelenkoberfläche ev. Freilegung des Knochens. Daß parallel damit die Veränderungen der einander zugekehrten Gelenkoberflächen gehen, ist selbstverständlich. Die verdickte Gelenkkapsel, speziell an der Plantarseite, sowie die allmähliche Schrumpfung der Ligamenta collateralia begünstigen die Beugekontraktur und machen sie mit der Zeit irreponibel.

Von der eigentlichen Hammerzehe zu trennen ist der sog. Hammerzehenplattfuß (Pes malleus valgus [ $\mathrm{Nicoladoni}$ ). Es handelt sich hierbei um eine Flexionskontraktur im Metatarsophalangealgelenke der Großzehe und nicht im I. Interphalangealgelenke. Zwar wollen wir nicht mit $\mathrm{C}$. Hof f mann nur Flexionskontrakturen der zweiten Zehe als Hammerzehen bezeichnen, sondern Beugekontrakturen im I. Interphalangealgelenke. Deren Ätiologie und pathologisch-anatomisches Bild haben wir eben ausführlich besprochen.

Die Pathogenese des Hammerzehenplattfußes ist scharf zu trennen von der Hammerzehe. Der Hammerzehenplattfuß ist nach $\mathrm{Nicoladoni}$, der ihn zuerst genauer untersucht und beschrieben hat, und $\mathrm{V}$ ulpi us eine Kontraktur infolge schmerzhafter Narbe am inneren Fußrande (Sensenhieb unterm inneren 
Knöchel), oder infolge Sehnenverletzung (Zerreißung der Plantarfascie) bei Klumpfußredressement mit konsekutiver Kontraktur. C. Hoffma $n$ bezcichnet ihn daher als erworbene fixierte Entlastungsstellung als folge eines schmerzerzeugenden Zustandes (Borchardt). Durch die dauernde fixierte Plantarkontraktur der Großzelle, meint $\mathrm{Nicoladoni}$, wird der innere Fußrand und mit ihm der innere lußbogen grehoben, der äußere aber, der von Anbeginn an fast aussschließlich zum Tragen verwendet wird, überlastet; in den I'ubertïtsjahren ist dieses nicht gleichgültig.

Der überladene äuBere Fubbogen sinkt ein, und damit ist der crste Anstob zum P'es planus gecreben. Rubritius nahm an, dabs erst der Plattful. bestehe und sodann durch Verlegung des Fußgewölbes nach vorn dic Zehendeformität sich ausbildet. Die gleiche Auffassung teilen Eichenwald, Kirsch und Ranneft. Durch die Zehenkontraktur will der Patient den schmerhaften Ilattul aufheben. Die Ïtiologie des Hammerzehenplattfuloses ist somit, obgleich in zwei Anschaungen geteilt, grundverschieden ron derjenigen der Hammerzche. Daher sind auch diese zwei Iseformitaten scharf auscinanderzuhalten.

J)ie: Iammerzehe ruft in manchen Fällen starke Schmerzen hervor beim (iehen und Stehen. IJer chronische Reiz des Schulwerkes macht sich immer aufs neue bemerkbar. Bl u mertrat die Anschanumgr, daß3 die hypertrophisch werdenden Nerven in ihren Verdickungen druckempfindlich seien. Oft strahlt der Schmerz weit nach oben kniewärts aus. Hinken ist die Folge. so berichtet l'etersen von einem lalle, wo ihm von einem Vater dessen 16 jähriger Junge zugeführt wurde wegen hoch. gradiger Schmerzen bei Hammerzehe. Der Vater stellte sogar die Amputation der Zehe anheim. I)annit soll wohl nur illustriert werden, wie grob die Beschwerden gegebenenfalls werden können.

1) ie Behandlung der Hammerzehe hann präventiv oder aber kurativ scin. Im letzteren Falle kann sie nur orthopädisch oder chirurgisch-orthopädisch scin. I)aß man, wie L a ncercaux es vorschlug, durch Jodkaligaben (per os oder extern) etwelchen Erfolg erzielen kann, ist unwahrscheinlich. Selbst Massage allein wird nicht genügeu, trotzdem durch dieselbe eine langsame Deh- 
nung der kontrakten Ligamenta collateralia und Gelenkkapsel möglich wäre.

Präventiv wird man auf richtig passende Schuhe sein Hauptaugenmerk zu richten haben. $\mathrm{Zu}$ hohe Absätze mit sehr schräg gestellter Fußachse begünstigen, wie wir gesehen haben, ebenfalls die Hammerzehenibildung. Sie sind daher zu bekämpfen. Die Fußachse im Schuh verlaufe möglichst horizontal, ev. nach vorn. etwas nach unten geneigt. Selbst kleine, unbedeutende Wunden der Fußsohle erfordern sorgfältige, antiseptische Behandlung zwecks Verhütung von schleichenden Phlegmonen mit konsekutiver Schrumpfung der Plantarfascie und der Ligamenta collateralia, sowie von kontrakten Hautnarben.

Rein orthopädisch behandelt werden die Hammerzehen mittels Einschubsohlen. Thilo gab Rindsledersohlen an, bei denen seitlich von der Hammerzehe zwei Längsspalten angebracht sind. Durch diese wird ein Flanellzügel durchgezogen, welcher über der gestreckten Hammerzehe (mit gepolsterter Unterseite) fixiert die Korrektur garantiert. P ürkhau er gab einen ähnlichen Apparat an, durch den die Hammerzehe mittels kleiner, auf der Ledersohle adaptierter Gurten fixiert wird. Durch Gipsverband hat man dauernde Extension und Korrektur der Deformität zu erzielen versucht. Weil zu umständlich, ist diese Methode nicht zur Geltung gekommen.

Die kurative Therapie kann nur chirurgisch sein. Alle Methoden basieren auf den anatomischen Veränderungen, die wir bei dieser Flexionskontraktur antreffen und oben bereits erörtert haben. Aus der Art der Bewertung der zur Kontraktur beitragenden Momente erklärt sich die Verschiedenheit der vorgeschlagenen Methoden. So empfahl Kö $\mathrm{n}$ i g die offene Durchschneidung aller Weichteile bis ins Gelenk: Haut, Beugesehne, Gelenkkapsel an der Plantarseite der Zehe, bis dieselbe gestreckt werden kann. Für 3-4 Wochen wurde dann die Zehe in Streckstellung fixiert. $\mathrm{König}$ berichtet von guten Resultaten. Petersen schildert eine analoge Methode folgendermaßen: „Ich durchschnitt an der Beugeseite entsprechend dem l. Interphalangealgelenke quer zunächst die Haut, dann die Beugesehne, schließlich die Gelenkkapsel, bis ich imstande war, die Zehe vollständig gerade zu stellen. Dabei liegen die Gelenkknorpel frei zutage. Die Haut- 
wunde klaffte ungefähr $2 \mathrm{~cm}$ weit bei gestreckter Stellung der Zehe. Anlegen eines Sche de schen Verbandes. Nach 3 Wochen ist die Wunde vollständig geheilt mit normaler Stellung der Zehe. $\mathrm{H}$ offa empfahl forciertes Redresscment und Festbandagieren der Hammerzehe mittels Heftpflasterstreifen. Im übrigen empfiehlt Hoffa das Vorgehen von Petersen, weil dem rein orthopädischen Verfahren weit überlegen. Martin gab zur Garantierungr des blutigen Redressement folgenden Verband an: Ein schmaler, einer Stauungsbinde entnommener Gummistreifen wird derart durch die Zehen geflochten, daß er an die Oberseite der Hammerzehe und Unterseite der übrigen Zehen zu liegen kommt. Die freien. Enden werden in Spiraltouren um FuB und Unterschenkel geführt und mit einer Binde festgewickelt. Hoff a hat damit wenig Erfolg erzielt.

Unter den chirurgischen Methoden, welche nicht, wie die bereits erwähnten, einfach alle spannenden Weichteile bis zum Gelenk durchtrennen, also eine Art blutiges Redressement darstellen, müssen wir diejenigen, die durch bloße Tenotomie die Kontraktur beseitigen wollen, von denen trennen, die noch Resek. tion der entsprechenden Gelenkenden hinzufügen.

So empfahl Bo y e r erst die bloße Durchtrennung der Strecksehnen. Später verlangte er die Exzision eines $11 / 2 \mathrm{~cm}$ langen Stückes der Extensorensehne zwecks Verhütung der Wiedervereinigung der Sehnenstümpfe. Cohen widersprach dieser Therapie, indem er richtigerweise die Kontrakturen der Sehnen als Folge, nicht als Ursache der Krankheit ansah. Durch bloße Durchtrennung der Extensorensehnen werde die Flexorenwirkung nur verstärkt. Blandin glaubte durch bloße Durchtrennung der Aponeurose Heilung zu crzielen. Von ähnlichen Gesichtspunkten ausgehend empfahl Cloqu ue die Durchtrennung der Bänder, die sich in der Plantaraponeurose kontrakt gebildet hatten. Elsberg empfahl mit Adams die subkutane Durchtrennung der Seitenbänder.

Für Fälle, wo bereits Ankylosierung im I. Interphalangealgelenke eingetreten ist, genügt die bloße Tenotomie nicht. Daher traten rivalisierend neben der bloßen Tenotomie noch Methoden auf den Plan mit Gelenkresektion. So empfahlen Karewski und Nasse die Resektion des Metatarso-Phalangealgelenks. Phil. 
Hof $\mathrm{fmann}$ macht einen bogenförmigen Schnitt in der Planta auf die Köpfchen der Metatarsi, legt dieselben frei durch Zurückpräparieren des Hautlappens. Ergiebige Resektion der Capitula ermöglicht Redressement in normale Stellung ohne Tenotomie. Seine Resultate bezeichnet er als überraschend gute. C. H of f mann empfiehlt mit $\mathrm{Nicoladoni}$ die Resektion der Grundphalanx, indem die Metatarsalköpfchen einen zu wichtigen Stützpunkt für den Fuß darstellen. Coutea ud empfahl für leichte und mittelschwere Fälle eine keilförmige Exzision der Phalanx, bei schweren Fällen unter gleichzeitiger Durchtrennung von Beuge- und Strecksehne. In schweren Fällen resezierte Cout e a u d von cinem Iorsalschnitte aus -- um eine störende Plantarnarbe zu verhüten, wie er sich ausdrückt -.. mit Giglisäge die Enden des verkrümmten Gelenks unter Tenotomie der Beugesehne. In manchen Fällen genügte die Resektion eines der beiden Gelenkenden, ohne Sehnendurchtrennung. O'Neill interponiert nach der Resektion des am meisten veränderten Gelenks subkutanes Fettgewebe. van Dam berichtet über gute Erfolge an der La n z schen Klinik durch Resektion im I. Interphalangealgelenke, oder Exartikulation der Endphalanx. Merril empfiehlt ein plastisches Verfahren, das in der queren lesektion der Trochlea der I. Phalanx und der Basis der II. Phalanx der deformen Zehe besteht und der Verlegung der Insertion der. Beugeseline an die Basis der I. Phalange und der Strecksehne ans Köpfchen des Metatarsus. Auch Soule empfiehlt die Resektion im I. Interphalangealgelenke von einem plantaren Schnitte aus. Terrier operiert erst nach Verheilung ev. Geschwüre. Denn aseptische Wundheilung ist ihm Vorbedingung zum Erfolge. Dorsal wird ein ellipsoides Stück Haut mitsamt dem Hühnerauge und dem darunterliegenden Schleimbeutel entfernt. Das Gelenk wird eröffnet nach Durchtrennung der Strecksehne, sowie der seitlichen Ligamenta collateralia. Die beiden Gelenkflächenenden werden mit schneidender Knochenzange reseziert, so daß die Zehen vollständig redressiert werden können. Weichteilnaht. Besonderes Augenmerk wird dem Verbande gewidmet: Eine mit Jodoformgaze gepolsterte Holzschiene kommt auf die Plantarseite der Zehe. Fixation mittels Binden. Nach 8 Tagen: Entfernung der Nähte. Bei prima intentio nochmals gleicher Verband für 
8 Tage. Nach 2 Wochen steht Patient auf und geht beschwerdefrei umher. Péraire empfiehlt das Vorgehen von Terrier. Nur rät er zur lintfernung des Nagels, indem in zwei Fällen nach der Operation trophische Störungen sich einstellten. Ähnlich wie Terrier ist das Vorgehen von Whe ele r unter Lokalanästhesie ausgeführt: Hautschnit, gleich wie Terrier unter Entfernung des Ilühnerauges bis aufs (iclenk; dabei Durchtrennung der Strecksehne über dem Gelenke. Mit dem Knochenhebel wird das Köpfchen der Grundphalanx emporgehoben und entfernt. Die Zehe läß3t sich nummehr leicht strecken und wird in Streckstellung durch Naht der Iautränder gehalten. Kollodiumverband. Durch entsprechend angelegten Heftpflasterstreifen wird die streckung der Zehe verhindert. Der Patient kann sofort herumgehen. S $\$$ mith ist warmer Anlä̈nger dieser Isthode; nur fügt er dic: Tenotomie der Beugeschne hinzu. Borchardt macht auch die Rescktion von einem I)orsalschnitte aus unter Exstirpation des Schleimbeutels. Ausgiebige Resektion macht nach ihm Tenotomice überflüssig.

I) ie auf Reselstion abziclenden Methoden nehmen den Hauptplatzein in der Therapie der Hammerzehe, und zwar die Resektion eines oder beider Gelenkenden des deformen I. Interphalangealgelenks. Wir verwenden diese Methode nur in Ausnahmefällen: denn in der zröß3ten Mehrzahl der Fälle erwies sich uns nachfolgende cinfache, von Herm Gelhcimrat P' a y r eingeführte Methode als die der Wahl.

Vorgängrig der (Jperation müssen er. Wunden und Geschwüre, sowic Hautekzeme beseitjgt werden; denn primäre Wundheilung ist Vorbedingung des Erfolges. Der Eingriff wird in Lokalanästhesic vorgenommen: Spiralschnitt auf der Plantarseite der kontrakten Zehe über II. Phalanx, I. Interphalangealgelenk und (irundphalanx, wie ich es in einer früheren Arbeit be. schrieben habe (Fig. 2) (siche: Zentralblatt für Chirurgie I9I7, Nr. 36), bis auf die Beugesehne. Mit scharfen Häkchen werden die Wundränder auf beiden Seiten auseinandergezorgen. Unter seitlicher Verziehung der Ieugesehne werden nun die seitlichen Partien des I. Interphalangealgelenks scharf freigelegt. Die Ligamenta collateralia, die frei daliegen, werden beiderseits vollständig reseziert. In der Mehrzahl der Fälle tritt nun plötzlich völ- 


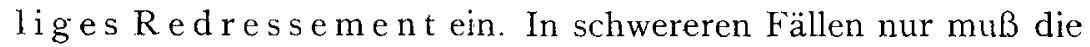
kontrakte Gelenkkapsel auf der Plantarseite noch eingeschnitten werden; alsdann ist das Redressement ebenfalls frei. Eine einzige gut adaptierte Hautnaht beschließt die Operation. Eng aneinanderliegende Nähte würden die Heilung nur erschweren. Die Zehe wird durch steril gepolsterte kleine Holzschiene von der Breite der Zehe, die auf der Plantarseite mittels zirkulärer Heftpflasterstreifen an die Zehe in Streckstellung geheftet wird, für 8 Tage fixiert gehalten. Hernach genügt nach Entfernung der Naht einfacher Schutzverband. Der Patient steht auf; die Stellung der Zehe ist normal. Wichtig ist, daß die plantarwärts verlager-

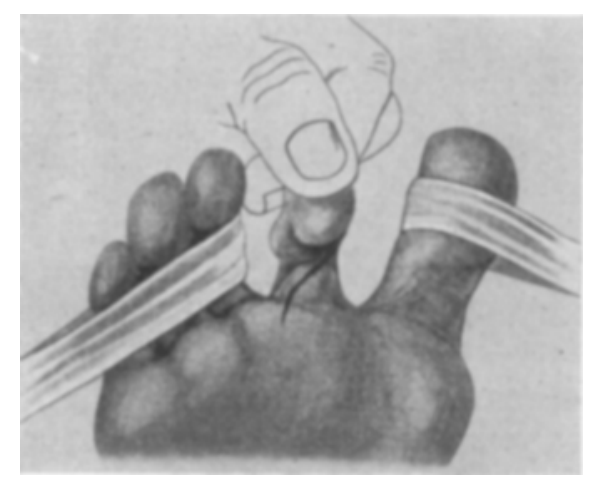

Fig. 2.

ten Ligamenta collateralia völlig reseziert werden. Bei der Anatomie sahen wir, daß die Hauptfasern dieser Ligamente hinter oder plantarwärts von der Längsachse durch die Zehenmitte verlaufen (vgl. Fig. I). Das leichte Redressement, das wir nach Resektion derselben beobachten, beweist die Bedeutung ihrer Kontraktur für die Genese der Hammerzehe. Bei hochgradigen Kontrakturzuständen derselben beobachtet man sogar dorsale Subluxationsstellung im I. Interphalangealgelenke. Dasselbe tritt auch ein bei brüsken Redressementversuchen. Dabei wirkt das distale Ende der Grundphalanx als Hypomochlion. Die Ligamenta collateralia, die sich stark anspannen, sind die Ursache.

Nur in veralteten Fällen mit stark deformen Gelenken und dementsprechend sekundär stark kontrahierten Beugesehnen ge- 
genügt das oben geschilderte Verfahren nicht. Da muß die Beugesehne in Z-Form plastisch verlängert werden, durch frontale Durchtrennung derselben und exakter Naht in Streckstellung der Zehe.

$\mathrm{Zu}$ Resektionen der Gelenkenden bei Hammerzehe waren wir nie genötigt.

Der große Vorteil des Spiralschnittes erhellt aus der freien Übersicht über das Operationsfeld, die man ohne nennenswerte Läsion erzielt. Aus der plantaren Lage des Schnittes erwächst keine Störung in der Wundheilung, vorausgesetzt, daß die Haut zweckentsprechend vorbereitet worden ist.

Die von Herrn Geheimrat Payr angegebene Methode der Hammerzehenoperation empfiehlt sich durch ihre Einfachheit für die meisten Fälle von Hammerzehe. Durch dieselbe wird innerhalb kurzer Zeit ein oft jahrlanges Leiden zur sicheren Heilung geführt.

Präventiv gegen Hammerzehen ist auf gutsitzendes Schuhwerk zu halten, welches das Abrollen des Fußes beim Gehen ohne Kompression der Zehen gestattet. Die Fußlängsachse soll nahezu horizontal verlaufen, ja nicht steil schräg von hinten oben nach vorn unten, wie es bei gewissen, früher schon gerügten Frauenschuhen mit hohen Absätzen der Fall ist. Der etwas schmale Schuh ist viel unschädlicher, als der selbst genügend breite mit überhohen Absätzen (P a y).

\section{Literaturverzeichnis.}

I. Adams, On contractions of the fingers and on hammertoe. London 1892.

2. - On the successful treatments of hammertoe. London 1888.

3. Anderson, Hammertoe. Clinical society. May I887.

4. --, Über die Pathologie u. Therapie der Finger- u. Zehenkontrakturen. Wien. med. Blätter I89I, 29-3I.

5. B ec, De l'orteil en marteau. Clin. franç. I89 I, 7 .

6. Bilhaut, De l'orteil en marteau. Clin. franç. 189I, 5 .

7. B landin, Nouveaux éléments d'anatomie descriptive. I837-38.

8. Blum, De l'orteil en marteau. Bull. de la soc. de chirurgie I883.

9. B orchardt, Chirurgie des Fußgelenkes u. des Fußes in v. Bruns-

Mikulicz Handb. d. prakt. Chir. 19I4, 5. Bd., 4. Aufl.

ıo. Boyer, Traité des maladies chirurgicales p. 1087. 
Ir. Chlumsky, Behandlung der Hammerzehe. Zeitschr. f. orth. Chir. Bd. 3I, S. 64 .

12. Cohen, Eug èn e, Orteil en marteau. Thèse. Paris 1887.

13. Couteaud, Etude sur l'orteil en marteau. Rev. de chir. 1908. Ref. Zentralbl. f. Chir. I908, Nr. 52.

I4. D a m, M. J. va n, Hamerteenen. Neederl. Tijdschr. v. Geneeskunde 1908, Nr. 18. Ref, Zentralbl. f. Chir. 1908, Nr. 49.

15. Debaussaux, De l'ortcil en marteau. Recueil de mém. de méd. milit. 1879 .

I6. Dubreuil, Mal dorsal des orteils. Gaz. des hôp. I87o.

17. Elsberg, A., Uber einen Fall von Hammerzehe. Diss. Greifswald 1898.

I8. Ewald, Dic Hebelwirkung des Fußes, wenn man sich auf die Zehen erhebt. Arch. f. Phys. 59.

19. Fra no. Bull. de la société de chir. I854 (n. Cohen).

20. H of $f$ a, A. Lehrb. d. orth. Chir. I905, 5. Aufl.

2I. - u. B lenke, A., Die orthopäd. Literatur. Stuttgart I905 (vollst. Lit. bis 1905).

22. Hoffmann, C., Zur Entstchung u. Behandlung der Klumpzehen. Zeitschr. f. orth. Chir. Bd. 8.

23. H of $\mathrm{fmann}$, Phil, An operation for severe grades of contracted or clawed toes. Amer. Journ. of orthop. surg. 19I2, Vol. IX, Nr. 3.

24. J o a ch im st h a l, G. Handb. d. orthopäd. Chir. Jena 1905, Bd. 2, H. 2. 25. Kirsch, E., Hallux mall. (varus). Zentralbl. f. Chir. 1897, Nr. 13. 26. $\mathrm{M}$ a $1 \mathrm{~g}$ a ign e. Leçons d'orthop. $\mathrm{i} 862$.

27. Martin, E., Zur Behandlung der Zehenkontrakturen insbesondere der Hammerzehe. Zeitschr. f. ärztl. Fortb. 1908, Nr. I9.

28. M e rkel, Fr. Handb. d. topogr. Anatomie. Braunschweig 1907. .

29. M erril, J. W., Conservative operative treatment of hammertoe. Amer. Journ. of orth. surg. 1912, Vol. X, p. 262.

30. N élaton. Pathologie chirurgic. (n. Cohen).

3r. N etzler, Eine seltene Fußdeformität. Zentralbl. f. chir.-mech. Orth. Bd. 6, H. 2 .

32. $\mathrm{Nicoladoni}$ Über Zehenkontrakturen. Wien, med. Wochenschr. I $88 \mathbf{r}$, S. $5 \mathbf{r}$.

33. - Der Hammerzehenplattfuß. Wien. klin. Wochenschr. I895, S. I5.

34. O'N eill, An arthropl. operation for hammertoe. Journ. of the amer. med. assoc. Igri, Vol. LVII.

35. Pérair e, M., Cinq cas d'orteil en marteau. Rev. de chir. I895, Nr. 7 .

36. Petersen, l., Zur operativen Behandlung verkrümter Zehen. Arch. f. klin. Chir. Bd, 37 .

37. Pétrequin. Anat. méd. chir. 1844 .

38. Phocas, De l'orteil en marteau. Gaz. des hôp. 1892.

39. P ü $r k$ ha u e r, Zur Behandlung der Hammerzehe. Zentralbl. f. chirmech. Orth. IgII. 
40. Ranneft. Zeitschr. f. orth. Chir. Bd. 4 .

41. Roche, Sanson et Lenoir. Nouveaux éléments de frathol. chisurgic. T. V (n. Cohen).

42. Roussclot, La toilette des pieds $17(x)$ (n. Cohen).

4.3. R ubriti us, II., Hammerzchenplattfuß3 u. Klumpzehenplattfuß3. Beitr. z. klin. Chir. Bd. 66.

44. $\mathrm{S}$ a B. Bull. de la société des chir. $\mathrm{I} 88_{3}$ (n. Cohen).

45. $\mathrm{S} \mathrm{chwarz}, T$., De l'orteil en martcau. Disc. à la soc. de chir. de Paris. Bull. méd. I 888 .

46. $\mathrm{S} \mathrm{mith}$, Operation for hammertoe. Nedical Press 19Io, Jan. 19.

47. Soulc, R., Operation for the correction of hammertoe. New Yorls med. Journ. I910, March 26.

4S. Thilo, O., Einschubsohlen zur Geradrichtung der Zehen. Zeitschr. f. orth. Chir. Bd. 4 .

49. Till manns, H. Lehrb. d. spez. Chir. II. T. Leipzig Igrr.

50. Valat, Double orteil en marteau. Annal. d'orth. I 888.

51. Vulpius, O., Ein Fall von P'es malleus valg. Münchn. med. Wochenschr. 1896, Nr. 35.

52. Wh e e ler, Simple operation for hammertoe. Medical Press 19 ro. 